\title{
Voltammetry and Singlemolecule in Situ Scanning Tunnelling Microscopy of the Redox Metalloenzyme Human Sulfite Oxidase
}

Yan, Jiawei; Frøkjær, Emil Egede; Engelbrekt, Christian; Leimkühler, Silke; Ulstrup, Jens; Wollenberger, Ulla; Xiao, Xinxin; Zhang, Jingdong

Published in:

ChemElectroChem

Link to article, DOI:

10.1002/celc.202001258

Publication date:

2021

Document Version

Peer reviewed version

Link back to DTU Orbit

Citation (APA):

Yan, J., Frøkjær, E. E., Engelbrekt, C., Leimkühler, S., Ulstrup, J., Wollenberger, U., Xiao, X., \& Zhang, J. (2021). Voltammetry and Singlemolecule in Situ Scanning Tunnelling Microscopy of the Redox Metalloenzyme Human Sulfite Oxidase. ChemElectroChem, 8(1), 164-171. https://doi.org/10.1002/celc.202001258

\section{General rights}

Copyright and moral rights for the publications made accessible in the public portal are retained by the authors and/or other copyright owners and it is a condition of accessing publications that users recognise and abide by the legal requirements associated with these rights.

- Users may download and print one copy of any publication from the public portal for the purpose of private study or research.

- You may not further distribute the material or use it for any profit-making activity or commercial gain

- You may freely distribute the URL identifying the publication in the public portal 


\section{ChemElectroChem}

\section{Fhemistry Europe}

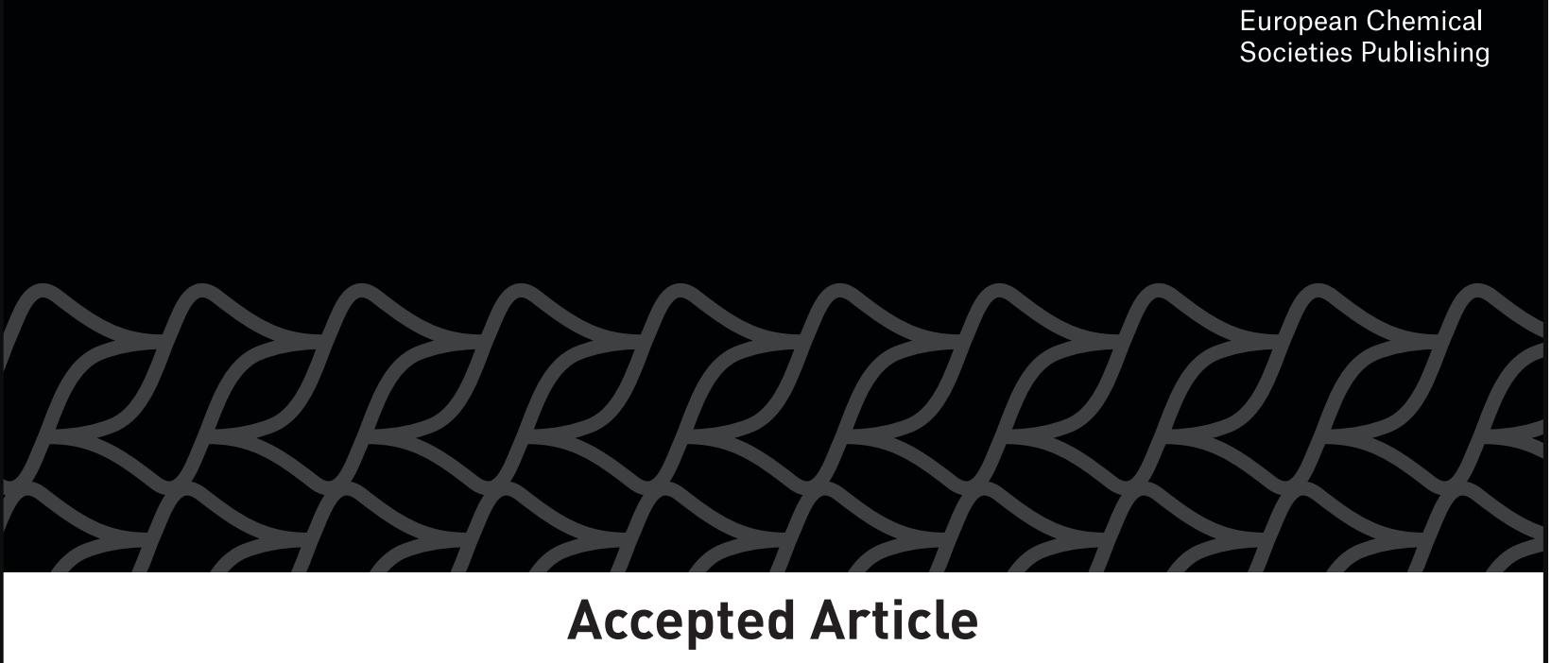

Title: Voltammetry and Single-molecule in Situ Scanning Tunnelling Microscopy of the Redox Metalloenzyme Human Sulfite Oxidase

Authors: Jiawei Yan, Emil Egede Frøkjær, Christian Engelbrekt, Silke Leimkühler, Jens Ulstrup, Ulla Wollenberger, Xinxin Xiao, and Jingdong Zhang

This manuscript has been accepted after peer review and appears as an Accepted Article online prior to editing, proofing, and formal publication of the final Version of Record (VoR). This work is currently citable by using the Digital Object Identifier (DOI) given below. The VoR will be published online in Early View as soon as possible and may be different to this Accepted Article as a result of editing. Readers should obtain the VoR from the journal website shown below when it is published to ensure accuracy of information. The authors are responsible for the content of this Accepted Article.

To be cited as: ChemElectroChem 10.1002/celc.202001258

Link to VoR: https://doi.org/10.1002/celc.202001258 


\title{
Voltammetry and Single-molecule in Situ Scanning Tunnelling Microscopy of the Redox Metalloenzyme Human Sulfite Oxidase
}

\author{
Jiawei Yan ${ }^{1,2}$, Emil Egede Frøkjar $^{l}$, Christian Engelbrekt ${ }^{l}$, Silke Leimkühler ${ }^{3}$, Jens Ulstrup ${ }^{1 *}$ ), \\ Ulla Wollenberger ${ }^{3}, X_{\text {inxin Xiao }}{ }^{1}$, and Jingdong Zhang ${ }^{1 \dagger}$
}

${ }^{1}$ Department of Chemistry, Technical University of Denmark, Building 207, Kemitorvet, DK2800 Kgs. Lyngby, Denmark

${ }^{2}$ State key Laboratory of Physical Chemistry of Solid Surfaces and College of Chemistry and Chemical Engineering, Xiamen University, Xiamen 361005, Fujian, P.R. China ${ }^{3}$ Department of Molecular Enzymology, University of Potsdam, 14476 Potsdam-Golm, Germany *)ju@kemi.dtu.dk

Prof. J. Yan

Mr. Emil E. Frøjkær

Dr. Christian Engelbrekt

Dr. Silke Leimkühler

Prof. Jens Ulstrup

Prof. Ulla Wollenberger

Dr. Xinxin Xiao

Prof. Jingdong Zhang

\begin{abstract}
Human sulfite oxidase ( $h \mathrm{SO})$ is a homodimeric two-domain enzyme central in the biological sulfur cycle. A pyranopterin molybdenum cofactor (Moco) is the catalytic site and a heme $b_{5}$ group located in the N-terminal domain. The two domains are connected by a flexible linker region. Electrons produced at the Moco in sulfite oxidation, are relayed via heme $b_{5}$ to electron acceptors or an electrode surface. Inter-domain conformational changes between an open and a closed enzyme conformation, allowing "gated" electron transfer has been suggested. We first recorded cyclic voltammetry (CV) of $h \mathrm{SO}$ on single-crystal $\mathrm{Au}(111)$-electrode surfaces modified by selfassembled monolayers (SAMs) both of a short rigid thiol, cysteamine and of a longer structurally flexible thiol, $\omega$-amino-octanethiol (AOT). $h \mathrm{SO}$ on cysteamine SAMs displays a well-defined pair of voltammetric peaks around $-0.207 \mathrm{~V}$ vs. SCE in the absence of sulfite substrate, but no electrocatalysis. $h \mathrm{SO}$ on AOT SAMs displays well-defined electrocatalysis, but only "fair" quality voltammetry in the absence of sulfite. We recorded next in situ scanning tunnelling spectroscopy (STS) of $h \mathrm{SO}$ on AOT modified Au(111)-electrodes, disclosing, a 2-5\% surface coverage of strong molecular scale contrasts, assigned to single $h \mathrm{SO}$ molecules, notably with no contrast difference in the absence and presence of sulfite. In situ STS corroborated this observation with a sigmoidal tunnelling current/overpotential correlation.
\end{abstract}




\section{Introduction}

Long-range electron transfer (LRET) between transition metal centres through protein "matter" is a core feature in photosynthesis and respiration, and in multi-centre redox enzyme catalysis ${ }^{[1-4]}$. LRET is often accompanied by features such as coupled proton electron transfer [5-8] " "gating"[3,4,9], and cooperative effects among different charge transfer steps ${ }^{[9,10]}$. The fundamental LRET phenomenon in large redox metalloprotein function is broadly understood, and exploited for example in electrochemical biosensors ${ }^{[11,12]}$ and biofuel cells, ${ }^{[13-15]}$ but conceptual and theoretical challenges remain and new challenges continue to emerge.

Molecular and biomolecular electrochemistry has developed as powerful tools in the mapping of adsorption, and of interfacial and intramolecular ET of immobilized redox metalloenzymes ${ }^{[16-17]}$. Electrochemical enzyme activity is exceedingly sensitive to the surface which is mostly tailored by self-assembled molecular monolayers (SAMs), where functionalized alkanethiols on gold electrode surfaces have been frequently used ${ }^{[18,19]}$. A long-standing issue is that the enzyme often shows no voltammetry itself, but binding of the enzyme substrate triggers strong electrocatalytic signals, indicative of conformational opening of productive interfacial and intramolecular ET channels between the metal redox centres and between the enzyme and an electrode surface ${ }^{[20-22]}$. The signals are associated with the enzyme itself, as the substrates (say dioxygen, nitrite, for laccase and nitrite reductase, respectively) exhibits no electrochemistry in these potential ranges. The opposite effect is also encountered. For example, ET between the two heme groups in the diheme protein cytochrome $c_{4}$ is not part of intermolecular ET between this protein and external reaction partners in solution, but a fast, sub-ms ET channel opens, when cyt $c_{4}$ is immobilized on well-defined SAM-modified $\mathrm{Au}(111)$-electrode surfaces ${ }^{[23]}$. Novel techniques and theory of electrochemical redox metalloprotein activity reaching even single-molecule resolution have been introduced over the last couple of decades ${ }^{[16]}$. Single-crystal, atomically planar electrode surfaces, and scanning tunnelling (STM) and atomic force microscopy (AFM), directly in aqueous biological media (in situ STM and AFM) have here been crucial. 

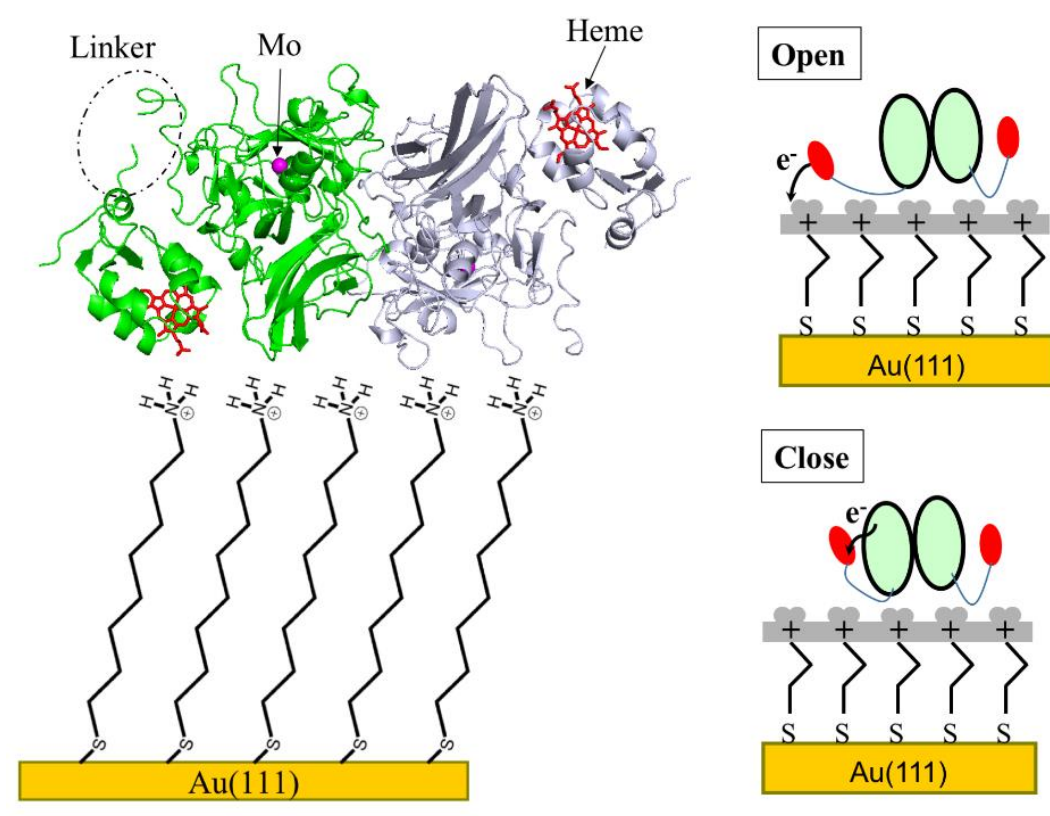

Figure 1 Left: Schematic view of $h \mathrm{SO}$ binding on electrode surface modified by a positively charged SAM. Right: Schematic view of switch between closed and open $h \mathrm{SO}$ conformations of electrochemical surface bound $h \mathrm{SO} . h \mathrm{SO}$ structure (PDB: 1SOX) is from ref.[27].

Human sulfite oxidase ( $h \mathrm{SO})$ is a dimeric two-domain metalloenzyme accommodating a pyranopterin molybdenum cofactor (Moco) in the central catalytic domain and a cytochrome $b_{5}$ type in the heme domain separated by a flexible ten-residue inter-domain polypeptide loop, Figure $1^{\text {[24-31] }}$. The catalyzed two-electron oxidation of sulfite to sulfate at the Moco centre is a terminal step in the biological sulfur cycle, while the heme domain relays electrons to an external electron acceptor, in nature cytochrome $c$, in bioelectrochemistry a solid electrode. ${ }^{[29-31]}$ Conformational switching between the open conformation that triggers ET between the heme group and the electrode, and the closed conformation that opens a gated intramolecular ET channel between the Moco and heme $b$ domains is a core step in the bioelectrochemical activity of the enzyme.

Facile direct interfacial electrochemical ET (DET), requires close distance between the negatively charged heme domain and positively charged SAM-modified electrodes, Figure 2. The electrostatic charge distribution at the surface around the catalytic Moco active site holds both positively and negatively charged residues, while the surface of the heme domain is largely negatively charged and both control binding of the Moco-domain and directs the heme group to the electrode surface via the flexible inter-domain linking chain, Figure 1. The surface potential distribution around both metallic centres thus highlight the importance of a positively charged 
electrode surface for favourable enzyme orientation. The sequential conformational, intramolecular, and interfacial ET steps was mapped in considerable detail recently ${ }^{[30,31]}$.
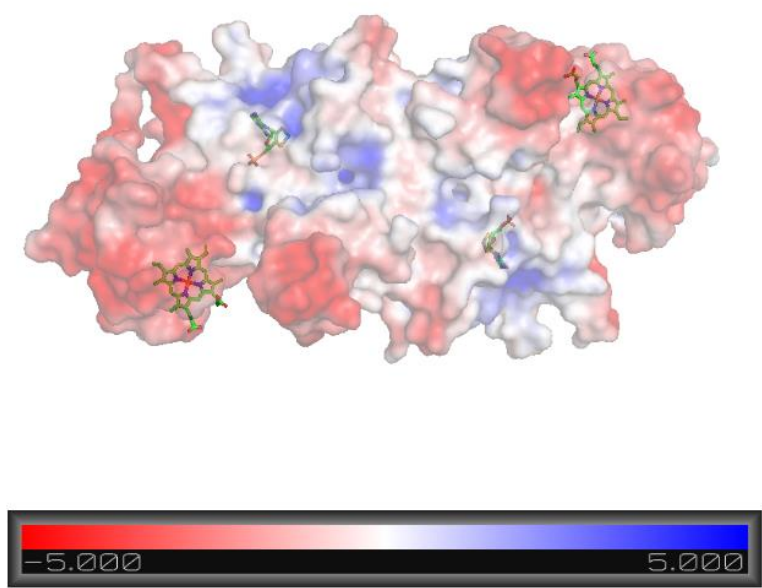

Figure 2 Electrostatic surface potential distribution of homodimeric chicken liver sulfite oxidase $^{[30,31]}$. Red and blue colour indicates negative and positive charges, respectively. The Moco and heme cofactors are indicated.

To retain gentle enzyme binding and at the same time high heme domain mobility in interfacial $h \mathrm{SO}$ electrocatalysis puts constraints on the SAM electrode modification. The two monomers in the $h \mathrm{SO}$ homodimer are held together by non-covalent, largely hydrophobic forces. The heme groups and their negatively charged local environments are, moreover, oppositely oriented. Whether the dimeric state of $h \mathrm{SO}$ is retained on exposure to strong electrostatic forces between a positively charged SAM modified electrode surface and the oppositely oriented negatively charged heme domains therefore remains a question ${ }^{[30,31]}$. It could be expected that $\omega$-terminated ammonium functionalized straight alkanethiol SAMs of a certain thickness (SAM molecular length) is the best compromise between a strongly positively charged surface to keep the $h \mathrm{SO}$ molecules gently immobilized in well-oriented surface orientation, at the same time ensuring enough SAM flexibility for the $h \mathrm{SO}$ gating step. Short SAM linkers would be too rigid, while longer SAM linkers attenuate electrochemical tunnelling unfavourably. Such considerations are the rationale for our particular SAM choice, borne out by comparison between a short rigid (cysteamine) and a longer, flexible linker unit ( $\omega$-amino octanethiol, AOT).

In previous bioelectrochemical studies chicken sulfite oxidase $(\mathrm{cSO})^{[24,28]}$ and $h \mathrm{SO}^{[30,31]}$ were immobilized on pyrolytic graphite, and on silver or gold electrodes modified with amino alkyl 
thiol SAMs or SAMs additionally covered with metallic nanoparticles. Common to these studies was the observation of a single pair of voltammetric peaks for the immobilized enzyme, which developed to a catalytic oxidation current on addition of enzyme sulfite substrate.

The first successful studies on direct ET were on chicken sulfite oxidase (cSO) on pyrolytic graphite $^{[24]}$ and SAM modified gold electrodes. ${ }^{[28]}$ The heme domain was suggested as an electronic relay between the catalytic site and the electrode in the catalytic turnover which was, however, much lower than for the enzyme in solution. Similar behaviour was found for the highly homologous $h \mathrm{SO}$. $h \mathrm{SO}$ and $c \mathrm{SO}$ have $68 \%$ sequence identity and $85 \%$ sequence similarity. ${ }^{[32]}$ Studies on $h \mathrm{SO}$ immobilized on amino terminated SAM modified silver electrodes using different ionic strength along with Raman spectroelectrochemistry and dipole moment calculations proved that the redox peaks originate from reduction and oxidation of the heme (FeIII/II), suggesting that the SO-dimer preferentially binds to the amino terminated SAM via the dimerization domain. ${ }^{[31-}$ ${ }^{33]}$ The mobility of the heme domain at high ionic strength ${ }^{[33]}$ and low viscosity ${ }^{[25,29]}$ facilitates the electrocatalytic reaction. An in-depth study of the transient response of the catalytic cycle of $h \mathrm{SO}$ immobilized supported by quantitative modelling, proved further that conformational changes between an open and a closed structure are indeed the limiting catalytic step of immobilized $h \mathrm{SO}{ }^{\left[{ }^{[31]}\right.}$ It remains, however, unclear if $h \mathrm{SO}$ retains homodimeric structure on the surface.

We describe here a combined electrochemical and single-molecule in situ STM/STS study of $h$ SO. The rationale for our choice of target enzyme is:

- The catalytic Moco reflects the challenging chemical properties of the element molybdenum with several oxidation states and ligand substitution processes involved.

- As in our electrochemical and single-molecule in situ STM/STS studies of the multi-centre blue copper enzymes, copper nitrite reductase (Achromobacter xylosoxidans) ${ }^{[21,34]}$ and laccase (Streptomyces coelicolor) $^{[22]}$, the enzyme substrate is a small molecule, i.e. sulfite. Binding of small substrate molecules to the enzyme are not structurally detectable by STM, but could induce changes in the electronic enzyme structure, reflected in conspicuous in situ STM/STS patterns.

- STM/STS in the electrochemically controlled in situ (in operando) mode offers two "spectral" features, i.e. the tunnelling current dependence of either the bias voltage, or of the overpotential ${ }^{[16]}$. As a second row transition metal with high electronic density, the $h$ SO Mo-centre would also offer prospects for strong in situ STM/STS contrast variation. 
- As noted, as a two-domain enzyme, a major enzyme conformational change from a "closed" to an open state, i.e. a "gated" ET step, Figure 1 is a controlling factor ${ }^{[24,30-32]}$. Intramolecular ET between the Mo-centre and the heme group triggered by sulfite binding, and gated ET between the heme group, and the electrode could offer intriguing in situ STM/STS patterns.

We have previously reported rationales for in situ STM/STS both of electrochemical ET of complex molecules and biomolecules ${ }^{[16]}$ and for single-molecule electrochemical "gating"[35]. In situ STM/STS of gated enzyme processes could offer new insight, now at the single enzyme molecule level. We first report $h \mathrm{SO}$ cyclic voltammetry (CV) on two $\omega$-amino-alkanethiol SAM modified single-crystal $\mathrm{Au}(111)$-electrode surfaces, chosen following our considerations concerning SAM charge and structural flexibility. This part offers a voltammetric prelude for the well-defined microenvironment in the second part of our study focussed on $h \mathrm{SO}$ in situ STM/STS. We conclude with a discussion of the observed in situ STM/STS sigmoidal tunnelling current/overpotential patterns and in situ single-molecule $h \mathrm{SO}$ electronic conductivity mechanisms.

\section{Experimental}

\subsection{Reagents}

hSO was expressed in Escherichia coli TP1000 cells containing plasmid pTG718 and purified as described. ${ }^{[36]}$ Aliquots of $39.2 \mu \mathrm{M} h \mathrm{SO}$ were stored in $0.01 \mathrm{M}$ phosphate buffer $(\mathrm{pH} 8)$ at $-20{ }^{\circ} \mathrm{C}$. Buffers were prepared from ultrapure $\mathrm{KH}_{2} \mathrm{PO}_{4}$ and $\mathrm{K}_{2} \mathrm{HPO}_{4}(\geq 99.999 \%$, Fluka) and sodium acetate (> $99 \%$, Sigma Aldrich), both pH 7.5. 8-amino-1-octanethiol ( $\geq 90 \%$, 8-AOT) was from Dojindo Laboratories and stored at $-20{ }^{\circ}$ C. Spectroscopy grade ethanol (Uvasol ${ }^{\circledR}$, Merck, $\geq 99.9$ $\%)$ was used for electrode cleaning. Sodium sulfite ( $\geq 98 \%$ ) was from Sigma-Aldrich. All reagents were used as received and stored in air-tight containers at temperatures as noted. Ultrapure water (Millipore Milli-Q, 18.2 M $\mathrm{cm}$ ) was used throughout.

\subsection{Electrochemical Electrodes and CV Instrumentation}

We used atomically planar single-crystal Au(111)-electrodes, in-house fabricated by Clavilier's method $^{[37]}$ and checked voltammetrically as described. ${ }^{[38]} \mathrm{CVs}$ were recorded using an in-house electrochemical cell with the SAM modified Au(111) working electrode, a freshly prepared 
reversible hydrogen electrode (RHE) as reference electrode, and a platinum mesh counter electrode, controlled by an AUTOLAB potentiostat (PGSTAT12, Switzerland). All glassware was boiled in $15 \mathrm{vol} \%$ nitric acid and rinsed with ultrapure water followed by sonication to remove all traces of nitric acid. All electrodes were annealed over a hydrogen flame prior to experiment. The RHE was calibrated vs. a saturated calomel electrode (SCE) after each experiment. Sample solutions were degassed by $5 \mathrm{~N}$ argon for half an hour prior to use and an argon atmosphere maintained throughout all experiments.

The clean $\mathrm{Au}(111)$-electrodes were submerged into solutions of $5 \mathrm{mM}$ cysteamine, or $3 \mathrm{mM} 8$ AOT in water for 24 hours at $4{ }^{\circ} \mathrm{C}$ and the SAM-modified electrodes rinsed thoroughly in Millipore water. $h \mathrm{SO}$ was immobilised onto the SAMs by soaking the rinsed electrodes in approximately 8 $\mu \mathrm{M}$ enzyme in Millipore water for 24 hours at $4{ }^{\circ} \mathrm{C}$.

\subsection{In situ Scanning Tunnelling Microscopy and Scanning Tunnelling Spectroscopy}

In situ STM imaging and in situ STS were recorded using a SPM 4500 instrument from Agilent Technologies combined with an in-house built teflon in situ STM cell with platinum wires as reference and counter electrodes. The STM electrochemical cell was boiled in 15 vol \% nitric acid solution before use. Platinum reference electrodes were calibrated against SCE.

W-tips for in situ STM/STS were fabricated by electrochemically etching tungsten wires following the method described by Ibe et al. ${ }^{[39]}$ The W-tips were coated by Apiezon wax to minimize the Faradaic current. Prior to each experiment, in situ STM/STS Au(111) electrode disk substrates were annealed at $860{ }^{\circ} \mathrm{C}$ for 8 hours followed by quenching in a $\mathrm{H}_{2}$ atmosphere. The constant current mode, rather the the constant height mode was used throughout.

\section{Results and Discussion}

No 3D structures of $h \mathrm{SO}$ is available, but a high-resolution structure for the homologous cSO is available $^{[27]}$ (PDB: 1SOX). As noted, $h \mathrm{SO}$ is an enzyme dimer in aqueous solutions, Figure 2 but the $h \mathrm{SO}$ aggregation state on surface immobilization remains unknown. As noted, the strongly negatively charged local protein surface environment of the two heme groups offers a rationale for gentle contact with positively charged SAMs, hence our choice of $\mathrm{H}_{3} \mathrm{~N}^{+}$-terminated alkanethiol SAMs. It is, further, often enough observed that the optimum length of SAM forming $\omega$ functionalized straight alkanethiols for redox protein immobilization must be chosen with 
conduct ${ }^{[40]}$. Long alkanethiols give small signals due to unfavourable tunnelling, while metalloprotein voltammetry at short alkanethiols are subject to interference from other elementary processes in the enzyme electrocatalysis.

We recorded $h \mathrm{SO}$ voltammetry on variable-length $\mathrm{H}_{2} \mathrm{~N}-\left(\mathrm{CH}_{2}\right)_{\mathrm{n}} \mathrm{SH}(\mathrm{n}=6,8,11$, and 16) SAMs. $\mathrm{H}_{2} \mathrm{~N}-\left(\mathrm{CH}_{2}\right)_{8}-\mathrm{SH}$ gave the best combination of robust and strong voltammetric signals, according with our discussion above and is our focus in the following. As a reference we recorded $h \mathrm{SO}$ voltammetry on $\mathrm{Au}(111)$ modified by the short rigid cysteamine SAM.

\section{1 hSO Voltammetry on Cysteamine and 8-Amino-1-octanethiol (8-AOT) SAM Modified} Single-crystal Au(111 electrodes)

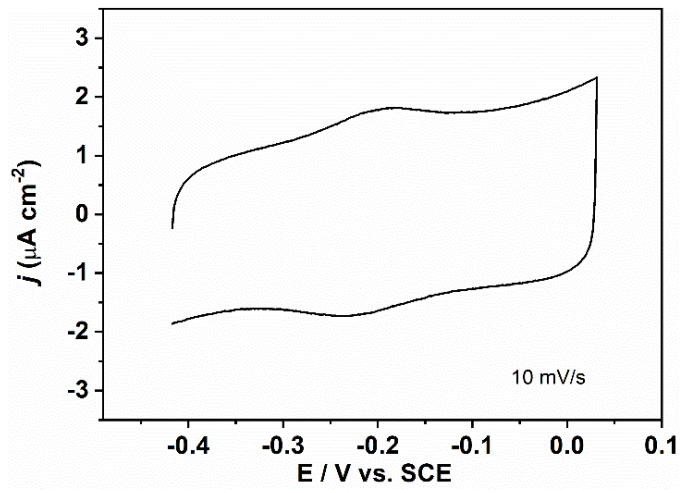

Figure 3 Cyclic voltammogram of $h \mathrm{SO}$ on $\mathrm{Au}(111)$-electrode surface modified by a cysteamine SAM. $0.02 \mathrm{M}$ phosphate buffer, $\mathrm{pH}$ 7. Scan rate: $10 \mathrm{mV} / \mathrm{s}$.

Figure 3 shows a representative $\mathrm{CV}$ of $h \mathrm{SO}$ in the absence of sulfite on a $\mathrm{Au}(111)$-electrode surface modified by a cysteamine SAM, showing a pair of well-defined peaks with a midpoint potential around $-0.207 \mathrm{~V}$ vs. SCE and a peak separation of $54 \mathrm{mV}$, which represents the heme group. ${ }^{[30,31,33]}$ The peak charges are $\mathrm{Q}_{\mathrm{a}}=260 \mathrm{nC}$, and $\mathrm{Q}_{\mathrm{c}}=250 \mathrm{nC}$ for anodic and cathodic peak current, respectively, leading to $Q_{a} / Q_{c}$ close to unity. As noted, well-defined enzyme voltammetry in the absence of substrate is unusual, cf. however, ref.[34] and here perhaps associated with the features of gated interfacial ET. The conspicuous capacitive background signal has also been observed elsewhere ${ }^{[40]}$ and reflects the composite dielectric properties of the heterogeneous and anisotropic molecular adlayers. No electrocatalytic voltammetry was observed for $h \mathrm{SO}$ on 
$\mathrm{Au}(111) /$ cysteamine SAMs in the presence of sulfite (data not shown). The reason for this observation remains unknown and is perhaps associated with freezing of the open/closed conformational change on such electrode surfaces. ${ }^{[41]}$ This pattern follows observations for cSO on cysteamine SAMs on polycrystalline gold electrodes. ${ }^{[32]}$
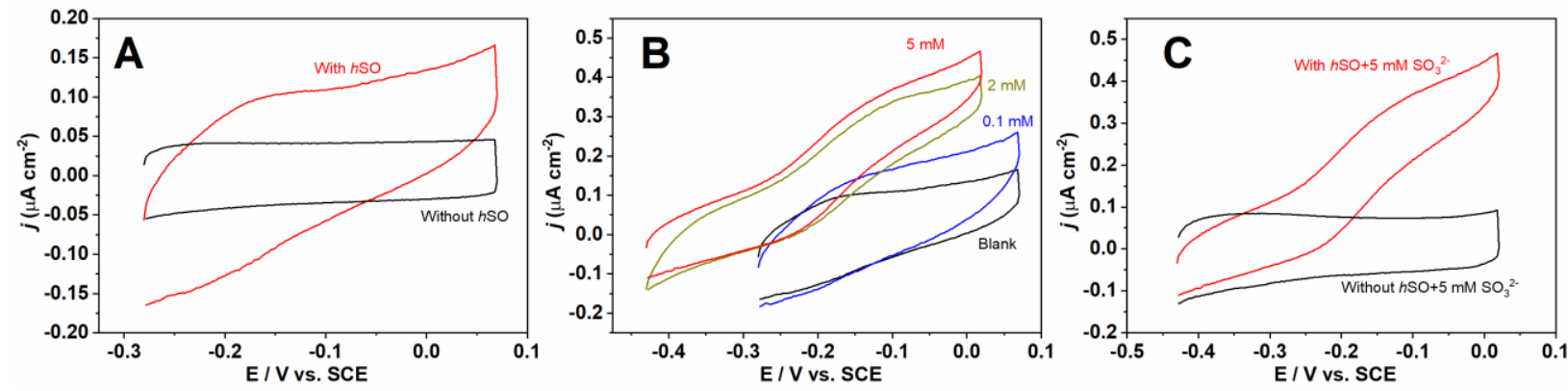

Figure $4 \mathrm{~A}$ : CVs of $\mathrm{Au}(111)$-electrode modified by an AOT SAM in the presence and absence of $h \mathrm{SO}$. No sulfite present; $0.02 \mathrm{M}$ sodium acetate medium, $\mathrm{pH}$ 7. B: CVs of $h \mathrm{SO}$ on $\mathrm{Au}(111)-$ electrode surface modified by an AOT SAM in the absence and presence of $0.1,2$ and $5 \mathrm{mM}$ sulfite as indicated. $\mathrm{C}$ : $\mathrm{CV}$ s of $\mathrm{Au}(111)$-electrode modified by an AOT SAM in the presence and absence of $h \mathrm{SO}$ with $5 \mathrm{mM}$ sulfite. All scan rates are $10 \mathrm{mV} / \mathrm{s}$.

As noted, 8-AOT SAMs on Au(111)-electrode surfaces give "fair" quality CVs, both with an anodic and a cathodic feature, in the absence of sulfite, as well as attractive electrocatalytic voltammograms (Figure 4), reflecting a good combination of positive surface charge densities, SAM structural flexibility, and facile interfacial ET. Figure 4A shows voltammetric signals for the $\mathrm{Au}(111)-8$-AOT SAM with and without immobilised $h \mathrm{SO}$ and no sulfite. The SAM alone without enzyme gives no voltammetric peaks. At a scan rate of $10 \mathrm{mV} / \mathrm{s}$, a pair of peaks with a midpoint redox potential of around $-0.179 \mathrm{~V}$ vs. SCE and a peak separation of $62 \mathrm{mV}$ is clearly visible for the $h \mathrm{SO}$ modified $\mathrm{Au}(111)-8$-AOT SAM, attributed to the heme domain and showing that the enzyme can be immobilized in an electrochemically functional state. The mid-point potential is close to the reported value of $-0.157 \mathrm{~V}$ vs. $\mathrm{Ag} / \mathrm{AgCl}(1 \mathrm{M} \mathrm{KCl})$ for $h \mathrm{SO}$ on indium tin oxide (ITO) modified with polyethylenimine (PEI)-entrapped CdS nanoparticles ${ }^{[42]}$, and $0.1 \mathrm{~V}$ vs. SHE for the same enzyme on gold nanoparticle modified electrodes ${ }^{[31]}$. The peak charges are $\mathrm{Q}_{\mathrm{a}}=18 \pm 3 \mathrm{nC}$, and $\mathrm{Q}_{\mathrm{c}}=3.3 \pm 0.4 \mathrm{nC}$ for anodic and cathodic peak current, respectively, or apparent surface coverages of $\Gamma_{\mathrm{a}}=2.6 \pm 0.4 \mathrm{pmol} / \mathrm{cm}^{2}$ and $\Gamma_{\mathrm{c}}=0.5 \pm 0.1 \mathrm{pmol} / \mathrm{cm}^{2}$. The surface coverage is low, corresponding to only a few percent $(2-5 \%)$ of a densely packed monolayer and accords with the 
coverage determined by in situ STM, Section 3.2, indicating that most or all of the enzyme molecules retain bioelectrochemical activity on immobilization. The coverages are notably lower than for the $\mathrm{Cu}$ - enzyme, nitrite reductase (Achromobacter xylosoxidans) on cysteamine modified $\mathrm{Au}(111)^{[21]}$, and laccase (Streptomyces coelicolor ${ }^{[22]}$ on variable-length alkanethiols. Unlike $\mathrm{Au}(111) /$ cysteamine/hSO, the large $\mathrm{Q}_{\mathrm{a}} / \mathrm{Q}_{\mathrm{c}}$ of ca. 5 indicates that the reductive step limits the overall ET, i.e. the rate of heme reduction is much lower than the rate of oxidation. Such a difference is most likely caused by intramolecular ET between Mo and heme $\mathrm{e}^{[30,41]}$, triggered by the conformational change of $h \mathrm{SO}$ as rate determining, but only on 8-AOT and not on cysteamine. $h \mathrm{SO}$ on the AOT modified electrode exhibits no catalytic behaviour in the absence of sulfite, but a well-defined electrocatalytic signal in the enzyme substrate saturation range around the heme group equilibrium potential in the presence of variable concentrations of $\mathrm{SO}_{3}{ }^{2-}$, Figure $4 \mathrm{~B}$. The catalytic response increases with increasing sulfite concentration and can be attributed to $h \mathrm{SO}$, as such a response is not obtained on a $\mathrm{Au}(111)-8$-AOT electrode without $h \mathrm{SO}$ (Figure 4C). The CVs suggest that $h \mathrm{SO}$ immobilization on 8-AOT SAMs offers good prospects for single-molecule in situ STM/STS of $h \mathrm{SO}$ on surfaces such as these, Section 3.2.

\subsection{In Situ Scanning Tunnelling Imaging and Spectrocopy of Surface Immobilized hSO}

Figures 5 and 6 show in situ STM images of $h$ SO immobilized on 8-AOT modified Au(111)electrode surfaces at different working electrode potentials and both in the absence and presence of sulfite substrate. The constant current mode was used throughout.

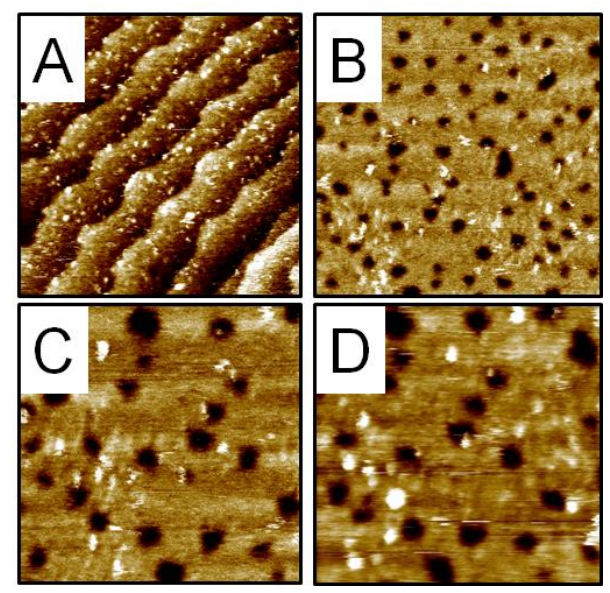

Figure 5 In situ STM images of $h \mathrm{SO}$ on $\mathrm{Au}(111)$-electrode surface modified by an AOT SAM. A) Without sulfite, $E_{w}:-0.26 \mathrm{~V}, E_{t i p}:-0.66 \mathrm{~V}, I_{t}: 0.04 \mathrm{nA}$, scan area: $50 \times 50 \mathrm{~nm}^{2}$. B) With $5 \mathrm{mM}$ sulfite, $E_{w}:-0.26 \mathrm{~V}, E_{t i p}:-0.66 \mathrm{~V}, I_{t}: 0.09 \mathrm{nA}$, scan area: $50 \times 50 \mathrm{~nm}^{2}$. C) With $5 \mathrm{mM}$ sulfite, $I_{t}$ : 
$0.09 \mathrm{nA}$, scan area: $\left.30 \times 30 \mathrm{~nm}^{2} . E_{w}:-0.26 \mathrm{~V}, E_{\text {tip }}=-0.66 \mathrm{~V} . \mathrm{D}\right)$ With $5 \mathrm{mM}$ sulfite, $I_{t}: 0.09 \mathrm{nA}$, scan area: $30 \mathrm{~nm}^{2} . E_{w}:-0.40 \mathrm{~V}, E_{\text {tip }}=-0.80 \mathrm{~V}$. $V_{\text {bias }}=E_{\text {tip }}-E_{\mathrm{w}}$, throughout. Potentials referred to SCE.

The $h \mathrm{SO}$ images without sulfite substrate, Figure 5A discloses a smooth background SAM, as common in thiol based SAMs with scattered black holes originating from the Au-S chemistry and $\mathrm{Au}$-atom extraction by interaction via the adsorbed thiyl linking in the SAM assembling process, leaving pits in nthe SAM (atomic scale "gold mining"[43,44] ). In addition, bright in situ STM contrasts are scattered over the surface. By their $\approx 4 \mathrm{~nm}$ size we assigned these to individual $h \mathrm{SO}$ molecules. As often observed for in situ STM of complex biomolecules the apparent vertical height, $\approx 0.25 \mathrm{~nm}$ is significantly smaller than the known structural dimensions of the biomolecular $\operatorname{targets}^{[45,46]}$. This discrepancy is rooted in STM recording the tunnelling conductivity through the target biomolecule, rather than the direct molecular structure. Smaller and rigid molecules show closer correspondence between real and apparent in situ STM heights. ${ }^{[47]}$ Figure 5A discloses other features. The surface population of bright molecular scale contrasts is not uniformly distributed over the electrode surface, but accumulate along step edges of the underlying $\mathrm{Au}(111)$-electrode surface. Also, and very notably, there is little if any contrast difference in the absence and presence of sulfite substrate. This is important in our discussion of the single-molecule $h \mathrm{SO}$ in situ STS mechanism below.

Figure 6 shows a sequence of $h \mathrm{SO}$ in situ STM images in a selected electrochemical overpotential range in the presence of $5 \mathrm{mM}$ sulfite. A stable, although small population of bright contrasts is apparent. The bright contrasts show systematic apparent height variation as the overpotential is varied across the equilibrium potential at constant bias voltage $(-0.4 \mathrm{~V})$, directly reflecting potential dependent $h \mathrm{SO}$ electronic conductivity, and in turn potential dependent single-molecule hSO ET activity. 


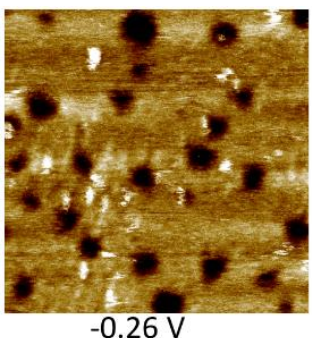

$-0.26 \mathrm{~V}$

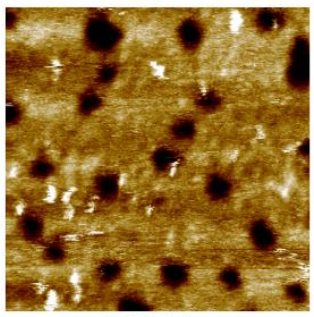

$-0.34 \mathrm{~V}$

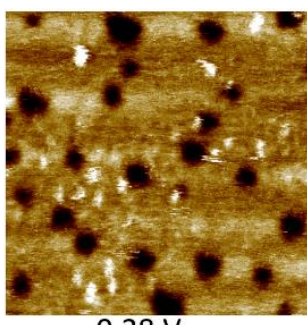

$-0.28 \mathrm{~V}$

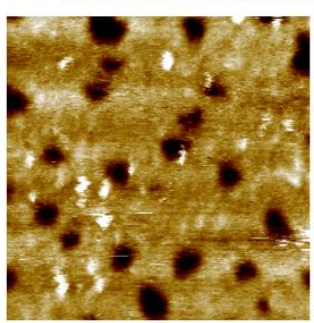

$-0.36 \mathrm{~V}$

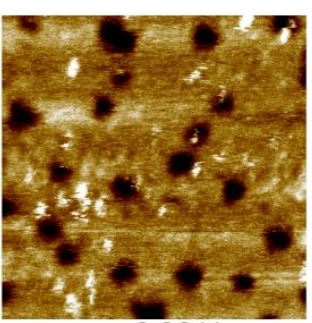

$-0.30 \mathrm{~V}$

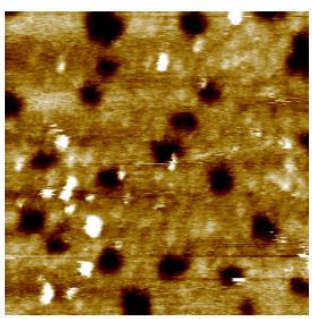

$-0.38 \mathrm{~V}$

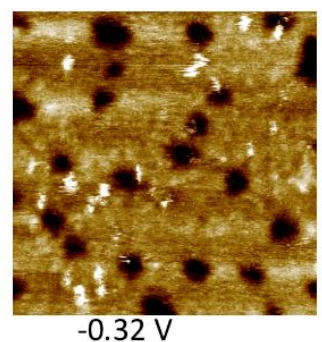

$-0.32 \mathrm{~V}$

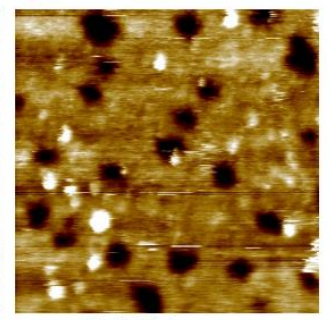

$-0.40 \mathrm{~V}$

Figure 6 In situ STM images of $h \mathrm{SO}$ on Au(111)-electrode surface modified by an AOT SAM. Scan area: $30 \times 30 \mathrm{~nm}^{2} 5 \mathrm{mM}$ sulfite. Selected potential values $E_{w}-0.26 \sim-0.40 \mathrm{~V}$. Bias voltage $V_{\text {bias }}=E_{\text {tip }}-E_{\mathrm{w}}=-0.4 \mathrm{~V}$ throughout. Constant current mode, $I_{t}: 0.09 \mathrm{nA}$. Potentials referred to SCE.

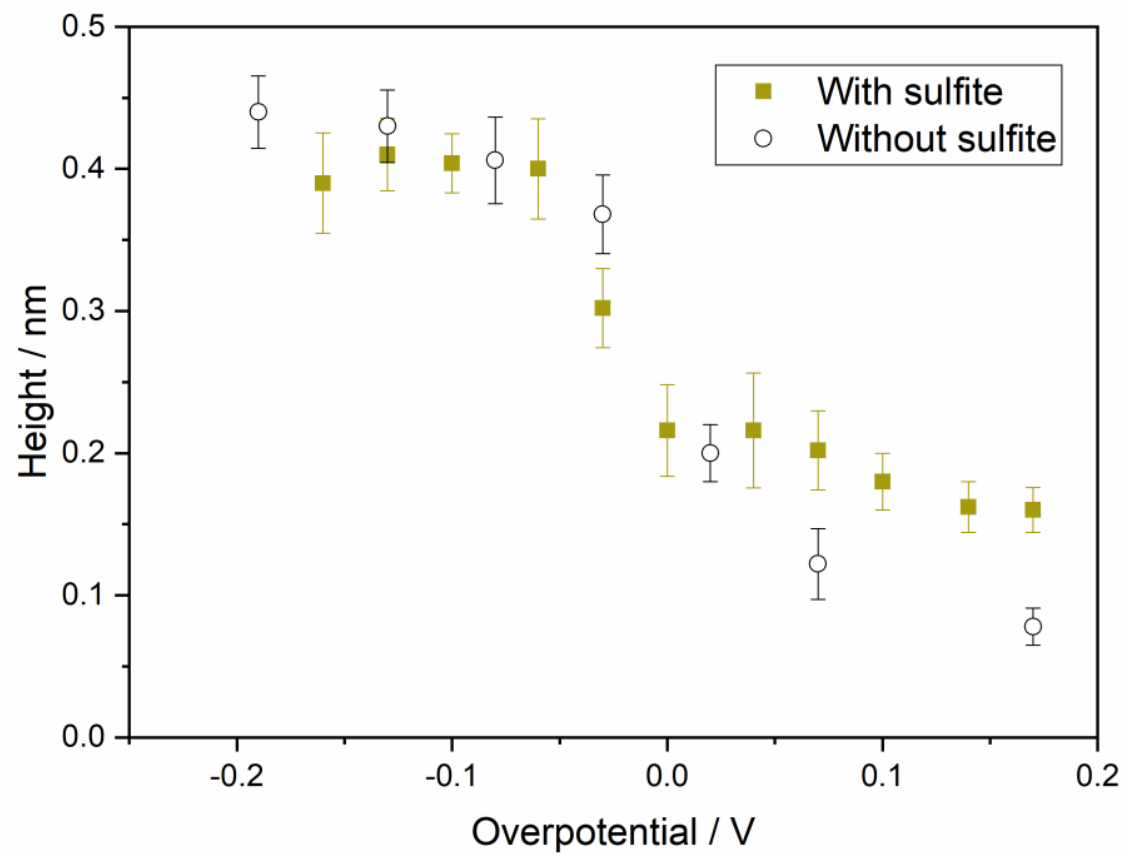

Figure 7 Apparent height variation with the overpotential of $h \mathrm{SO}$ on AOT SAM modified $\mathrm{Au}(111)$-electrode surfaces in the absence and presence of $5 \mathrm{mM}$ sulfite over the full overpotential range recorded. Constant current mode, setpoint current $0.09 \mathrm{nA}$. Bias voltage $-0.4 \mathrm{~V}$ throughout. Averaged over a number of $30 \times 30 \mathrm{~nm}^{2}$ frames and bright in situ STM surface contrasts as in 
Figure 6. The overpotential is defined as $E_{\mathrm{w}}-E_{\mathrm{mid}}$ where $E_{\mathrm{mid}}$ is the $h \mathrm{SO}$ midpoint potential of the cathodic and anodic peaks determined from Figure 4, both referred to SCE.

The potential dependent constant current in situ STM image contrast variation is recast in Figure 7, which essentially displays $h \mathrm{SO}$ bioelectrochemistry, now at the level of the single enzyme molecule both with and without $5 \mathrm{mM}$ sulfite present. The data points represent average apparent height values of the bright in situ STM contrasts over all the bright contrasts in a whole $30 \times 30$ $\mathrm{nm}^{2}$ frame at each overpotential value. We note first a clear transition from a low tunnelling current state at high potentials to a high tunnelling current state at low potentials. Single-molecule transition from a resting to an active enzyme state was observed in our previous studies of the blue multi-copper enzymes nitrite reductase $(\mathrm{CuNiR})^{[21]}$ and laccase ${ }^{[22]}$, but the $h \mathrm{SO}$ data, Figure 7 are different. Neither voltammetry nor in situ STM images could be observed for the blue Cu-enzymes in the absence of enzyme nitrite or dioxygen substrate, whereas substrate inlet triggered strong voltammetric signals and in situ STM contrasts. Secondly, the single-molecule constant current in situ STS of $h \mathrm{SO}$ also differs from constant current in situ STM/STS of the simpler ET metalloproteins azurin ${ }^{[16,45]}$ and cytochrome $b_{562}{ }^{[48]}$ as well as from simpler organic molecules (viologens) ${ }^{[46]}$ and transition metal complexes ${ }^{[49]}$. All of these display a strong in situ STS maximum around the equilibrium potential, whereas hSO displays an apparent sigmoidal pattern with a tunnelling current plateau.

The $E_{\mathrm{w}}$ value in Figures 5-7, - $-0.26 \mathrm{~V}(\mathrm{SCE})$ is about $80 \mathrm{mV}$ more negative than the voltammetric value from Figure 4 determined as $-0.179 \mathrm{~V}$ (SCE). The constant current in situ STM images were recorded in the potential range -0.34 to $-0.01 \mathrm{~V}$ (SCE) i.e. an overpotential range of about \pm 160 $\mathrm{mV}$, from which the data in Figure 7 was derived. With a view on the large negative bias voltage used the voltammetric midpoint potential and the midpoint potential in the sigmoidal-like constant current in situ STS cannot be expected to coincide exactly. As outlined in detail elsewhere. ${ }^{[16,49]}$, the target molecule in the in situ STM/STS configuration is thus exposed to an additional certain fraction of the bias voltage compared to the voltammetric configuration with just a single electrochemical surface.

Two particular features thus distinguish single-molecule $h \mathrm{SO}$ constant current in situ STS from single-molecule constant current in situ STS of other metalloproteins and metalloenzymes:

- hSO displays no constant current in situ STS maximum but a sigmoidal pattern. Nernstian redox equilibrated conductivity ${ }^{[50]}$ and "gated" tunnelling ${ }^{[35]}$ are relevant mechanistic rationales for a 
sigmoidal pattern, and the "flip-flop" mechanism of $h \mathrm{SO}$, with a conformational switch of the heme domain as a core step could favour a gated mechanism. A third rationale could, however, well be rooted in the large bias voltage needed $(-0.4 \mathrm{~V})$. Following reported observations on constant current in situ STS of transition metal complexes in ionic liquids ${ }^{[51]}$, and theoretical support $^{[52]}$, a mechanism of coherent, multi-ET is in fact likely to prevail in an intermediate overpotential range, before tunnelling current decline at high positive potentials take the redox level(s) to locations remote from the Fermi levels of the enclosing substrate and tip electrodes. A conspicuous maximum in the tunneling current/overpotential correlation is expected when the bias voltage, $V_{\text {bias, }}$ i.e. the quantity $\left|e V_{\text {bias }}\right|$ is small compared with the solvent reorganization free energy, $E_{\mathrm{r}}$, i.e. $\left|e V_{\text {bias }}\right|<(<) E_{\mathrm{r}}$. In the narrow tunneling gap $E_{\mathrm{r}}$ is small compared with the semiinfinite single-surface macroscopic electrochemical surface, say a value in the range of a few hundred $\mathrm{mV}$ or so. $\left|e V_{\text {bias }}\right|$ can therefore no longer be regarded as "small". A new overpotential independent multi-electron transfer in situ STM mechanism can then take over in an intermediate overpotential range as noted before the tunneling current drops to low values at higher positive overpotentials. This mechanism is a quite plausible origin of the plateau observed, as laid out in detail elsewhere ${ }^{[16,45,52]}$.

- A second major difference from the multi-copper enzymes is the virtually identical constant current in situ STM/STS pattern of $h \mathrm{SO}$ in the presence and absence of sulfite substrate. This unexpected observation must be associated with electron flow through the, free or substrate-bound enzyme rather than directly with enzyme activity. Electron flow through the enzyme must therefore prevail over substrate triggered enzyme conductivity in the in situ STM/STS process.

\subsection{Single-molecule hSO Mechanistic and Electronic Function, and Some Notes of Conclusion}

By its molecular structure and catalytic mechanism, sulfite oxidases are exciting enzymes. A Mopyranopterin centre with a range of oxidation states is the core of the structural enzyme features, and the gated "on-off" heme domain feature, well supported by mechanistic analysis, and in other ways are core elements in the electrocatalytic patterns ${ }^{[24-31]}$.

We first recorded $\mathrm{CV}$ of $h \mathrm{SO}$ at single-crystal, atomically planar $\mathrm{Au}(111)$-electrode surfaces variably modified by positively charged SAMs. With a view on the gated $h \mathrm{SO}$ activity we exploited both a short rigid SAM forming thiol, cysteamine, and a longer, structurally flexible SAM former, $\omega$-amine octanethiol (AOT). In accordance with the flexible communication 
between the catalytic Mo-domain and the electrochemically gated heme group domain, $h \mathrm{SO}$ immobilization on the cysteamine SAM displays good voltammetry but no electrocatalysis. In contrast, hSO immobilization on the AOT SAM surface gives conspicuous electrocatalysis but only "fair" voltammetry in the absence of sulfite substrate. This behaviour accords with the structurally "soft" $h \mathrm{SO}$ pattern but differs from the voltammetric behavior of the blue oxidases, for which no voltammetry is observed in the absence of nitrite or dioxygen substrate but strong electrocatatalytic signals appear as substrate is let in ${ }^{[21,22,53]}$.

Constant current in situ STM and STS of $h \mathrm{SO}$ is the second major outcome of our study. In situ STM imaging close to the equilibrium potential, both in the absence and presence of sulfite substrate, Figure 5 and 6 - is at the level of the single molecule, but the bright single-molecule scale contrasts prompt other observations. The molecular scale structures are not uniformly distributed over the surface but gather around the step edges of the underlying Au(111)-electrode surface, Figure 5. Further, the surface density estimated from the in situ STM images - is only about 2 pmol cm$~^{-2}$ or $2-5 \%$ of a dense monolayer for a molecular diameter of $6-8 \mathrm{~nm}$. This coverage accords with the coverage estimated from cyclic voltammetry, Figure 4, and suggests that all or most immobilized $h \mathrm{SO}$ molecules although sparsely populating the surface, have retained ET activity. In this respect $h \mathrm{SO}$ follows the much simpler cupredoxin azurin on variablelength alkanethiol SAMs, for which all immobilized azurin molecules were found to retain electrochemical activity ${ }^{[53]}$.

A further notable observation is that in situ STM/STS gives almost the same contrasts and in situ STS in the absence and presence of sulfite, Figure 7. This suggests that in situ STM/STS does not monitor single-molecule enzyme activity, but rather a tunnelling process through one or both of the enzyme domains, independently of sulfite. As noted, this is also quite different from the blue copper enzymes and must be rooted in the different molecular enzyme mechanisms for $h \mathrm{SO}$ and the copper enzymes.

The in situ STS pattern, Figure 7 underlines the distinct single-molecule $h$ SO electrochemical ET behaviour. The apparent tunnelling height variation with the overpotential shows a sigmoidal rather than a "spectroscopic" correlation as for simple ET proteins and smaller redox molecules. Simple Nernstian equilibrium ${ }^{[50]}$ or interfacial ET via a "soft" gating mode ${ }^{[35]}$ were noted as possible rationales, and "soft mode" gating would be attractive in view of the gating enzyme 
feature. Gating would then, however, have to apply to the tunnelling mechanism of both the enzyme itself and the enzyme in electrocatalytic action.

As finally noted, the large bias voltage needed ( $-0.4 \mathrm{~V})$ could well cause both a negative potential shift of the in situ STS feature compared with the voltammetric thresholds, and an intermediate plateau in the in situ STS profile, where coherent multi-ET prevails, before the current ultimately declines at still more negative overpotentials ${ }^{[52]}$. Such behaviour was reported for an osmium complex ${ }^{[51]}$, and it cannot be excluded that the tunnelling current plateau seen in Figure 7 reflects such an intermediate overpotential range and that the current would decrease at still larger negative overpotentials.

Overall our single-crystal and single-molecule electrochemical $h \mathrm{SO}$ study has disclosed intriguing electronic enzyme properties, of possible broader importance in pure and applied enzyme electrochemistry. A remaining, intriguing issue is the electronic origin of the bright in situ STM/STS contrasts. The contrasts are both of single-molecule scale and display a conspicuous in situ STS feature, but the resolution is not at a level, where for example monomer/dimer distinction of these soft materials is feasible. The core overpotential feature appears in the overpotential range -(0-80) $\mathrm{mV}$, or a working electrode potential range of $-(200-280) \mathrm{mV}$ vs. SCE, shifted by $\approx 100$ $\mathrm{mV}$ compared with the midpoint potential range determined voltammetrically. This is notable in view of the large negative bias voltage used $(-0.4 \mathrm{~V})$ and offers challenges as to the bias voltage distribution in the tunnelling gap. Also, the midpoint potentials of the heme $b_{5}$ and Moco $(\mathrm{Mo}(\mathrm{V}) /(\mathrm{VI}))$ centres determined spectroelectrochemically using a mini-grid Au-electrode are quite close, i.e. $62 \mathrm{mV}$ and $42 \mathrm{mV}$, respectively vs. $\mathrm{SHE}^{[54]}$, or -173 and $-193 \mathrm{mV}$, respectively vs. SCE. These are too close that in situ STS enables resolution into separate contributions from heme $b_{5}$ and Moco. It cannot be excluded, therefore that the observed in situ STS profile reflects a composite process, in which intramolecular ET between the Moco and heme $b_{5}$ domains is also involved. Tunnelling current/bias voltage correlations and possible current rectification ${ }^{[55]}$ may hold clues to this important issue.

\section{Acknowledgement}

Financial support from The Danish Council for Independent Research for the YDUN project (DFF 4093-00297) to JZ is gratefully acknowledged. JWY acknowledges financial support from Natural Science Foundation of China (No. 21673193). UW and SL gratefully acknowledge funding by the 
Deutsche Forschungsgemeinschaft (DFG, German Research Foundation) under Germany's Excellence Strategy-EXC 2008-390540038-UniSysCat.

\section{'Deceased}

Keywords: Cyclic voltammetry; Human sulfite oxidase; In situ scanning tunnelling spectroscopy; Self-assembled molecular monolayers; Single-crystal gold electrodes.

\section{References}

[1] H.B. Gray, J.R. Winkler, Biochim. Biophys. Acta - Bioenergetics 2010, 1797, 1563-1572.

[2] H.B. Gray, J.R. Winkler, Quart. Rev. Biophys. 2003, 36, 341-372.

[3] A.M. Kuznetsov, Charge Transfer in Physics, Chemistry and Biology, 1995, Gordon \& Breach. Reading.

[4] A.M. Kuznetsov, J. Ulstrup, Electron Transfer in Chemistry and Biology - An Introduction to the Theory. Wiley, Chichester: 1998.

[5] A.M. Kuznetsov, J. Ulstrup, Can. J. Chem. 1999, 77, 1085-1096.

[6] J.P. Klinman, A.R. Offenbacher, Acc. Chem. Res. 2018, 51, 1966-1974.

[7] S. Hammes-Schiffer, Acc. Chem. Res. 2009, 42, 1881-1889.

[8] A.M. Kuznetsov, J. Ulstrup, Russian J. Electrochemistry 2003, 39, 9-15.

[9] C.M. Paquete, D.L. Turner, R.O. Louro, A.V. Xavier, T. Catarino, Biochim. Biophys. Acta 2007, 1767, 1169-1179.

[10] L.S. Conrad, J.J. Karlsson, J. Ulstrup, Eur. J. Biochem. 1995, 231 133-141.

[11] F.A. Armstrong, H.A.O. Hill, N.J. Walton, Direct electrochemistry of redox proteins. Acc. Chem. Res. 1988, 21, 407-413.

[12] T. Siepenkoetter, U. Sala-Kosla, X. Xiao, S. Belochapkine, E. Magner, Electroanalysis 2016, $28,2415-2423$.

[13] J. Tang, X. Yan, C. Engelbrekt, J. Ulstrup, E. Magner, X. Xiao, J. Zhang, Bioelectrochemistry 2020, 134, 107537.

[14] X. Xiao, H.-Q. Xia, R. Wu, L. Bai, L. Yan, E. Magner, S. Cosnier, E. Lojou, Z. Zhu, A. Liu, Chem. Rev. 2019, 119, 9509-9558.

[15] D. Leech, P. Kavanagh, W. Schuhmann, Electrochim. Acta 2012, 84, 223-234. 
[16] J. Zhang, A. M. Kuznetsov, I.G. Medvedev, .Q. Chi, T. Albrecht, .P.S. Jensen, J. Ulstrup, Chem. Rev. 2006, 108, 2737-2791

[17] J.N. Butt, F.A. Armstrong, In: O. Hammerich, J. Ulstrup, Eds., Bioinorganic Electrochemistry, 2008, Springer, Dordrecht, pp. 91-128.

[18] J.R. Reimers, M.J. Ford, A. Halder, J. Ulstrup, N.S. Hush, Proc. Nat. Acad. Sci. USA 2016, 113, E1424-E1433.

[19] Q. Chi, M.J. Ford, A. Halder, N.S. Hush, J.R. Reimers and J. Ulstrup, Curr. Opin. Electrochemistry 2017, 1, 7-15.

[20] D. Zigah, E. Lojou, A. de Poulpiquet, ChemElectroChem 2019, 6, 5524-5546.

[21] J. Zhang, A.C. Welinder, A.G. Hansen, H.E.M. Christensen, J. Ulstrup, J. Phys. Chem. B 2003, 107, 12480-12484.

[22] V. Climent, J. Zhang, E.P. Friis, L.H. Oestergaard, J. Ulstrup, J. Phys. Chem. C 2012, 116, 1232-1243.

[23] Q. Chi, J. Zhang, T. Arslan, L. Borg, G.W. Pedersen, H.E.M. Christensen, R.R. Nazmutdinov, J. Ulstrup, J. Phys. Chem. B 2010, 114, 5617-5624.

[24] S.J. Elliott, A.E. McElhaney, C. Feng, J.H. Enemark, F.A. Armstrong, J. Am. Chem. Soc. 2002, 124, 11612-11613.

[25] C. Feng, R.V. Kedia, J.T. Hazzard, J.K. Hurley, G. Tollin, J.H. Enemark, Biochemistry, 2002, $41,5816-5821$.

[26] C. Kisker In: A. Messerschmidt, R. Huber, T. Poulos, K. Wieghardt, Eds., Handbook of Metalloproteins 2001, Wiley, Chichester, pp. 1121-1135.

[27] C. Kisker, H. Schindelin, A. Pacheco, W.A. Wehbi, R.M. Garrett, K.V. Rajagopalan, J.H. Enemark, D.C. Rees, D. C. Cell 1997, 91, 973-983.

[28] E.E. Ferapontova, T. Ruzgas, L. Gorton,.Anal.Chem. 2003, 75, 4841-4850.

[29] C. Feng, G. Tollin, J.H. Enemark, Biochim. Biophys. Acta 2007, 1774, 527-539.

[30] S. Frasca, O. Rojas, J. Salewski, B. Neumann, K.Stiba, I.M. Weidinger,B. Tiersch, S. Leimkuehler, J. Koetz, U. Wollenberger, Bioelectrochemistry 2012, 87, 33-41.

[31] T. Zeng, S. Leimkuehler, U. Wollenberger, V. Fourmond, J. Am. Chem. Soc. 2017. 139, 11559-11567.

[32] U. Kappler, J.H. Enemark, J. Biol. Inorg. Chem. 2015, 20, 253-264. 
[33] Sezer, M., Spricigo, R., Utesch, T., Millo, D., Leimkuehler, S., Mroginski, M.A., Wollenberger, U., Hildebrandt, P., Weidinger, I.M., Phys. Chem. Chem. Phys. 2010, 12, 78947903

[34] A.C. Welinder, J. Zhang, A.G. Hansen, K. Moth-Poulsen, H.E.M. Christensen, T. Bjørnholm, A.M. Kuznetsov, J. Ulstrup, Z Phys. Chem. 2007, 221, 1343-1378.

[35] W. Haiss, T. Albrecht, H. van Zalinge, S.J. Higgins, D. Bethell, H. Höhenreich, D.J. Schiffrin, R.J. Nichols, A.M. Kuznetsov, J. Zhang, Q. Chi, J. Ulstrup, J. Phys. Chem. B 2007, 111, 67036712.

[36] C.A.Temple, T.N. Graf, K.V. Rajagopalan, Arch. Biochem. Biophys. 2000, 383, 281-287.

[37] J. Clavilier, R. Fauré, G. Guinet, R. Durand, J. Electroanal. Chem. 1980, 107, 205-209.

[38] Q. Chi, J. Zhang, E.P. Friis, J.E.T. Andersen, J. Ulstrup, Electrochem. Comm. 1999, 1, 91-96.

[39] J. P. Ibe, P. P. Bey, S. L. Brandow, R. A. Brizzolara, N. A. Burnham, D. P. DiLella, K. P. Lee, C. R. K. Marrian, R. J. Colton, Journal of Vacuum Science \& Technology A 1990, 8, 3570-3575.

[40] Q. Chi, J. Zhang, J.E.T. Andersen, J. Ulstrup, J. Phys. Chem. B 2001, 105, 4669-4679.

[41] S. Ma, C.V.F.P. Laurent, M. Meneghello, J. Tuoriniemi, C. Oostenbrink, L. Gorton, P.N. Bartlett, R. Ludwig, ACS catalysis, 2019, 9, 7607).

[42] T. Zeng, S. Leimkuehler, J. Koetz, U. Wollenberger, ACS Appl. Mater. Interf. 2015, 7, 2148721494.

[43] Y. Wang, Q. Chi, N.S. Hush, J.R. Reimers, J. Zhang, J. Ulstrup, J. Phys. Chem. C 2011, 115, 10630-10639.

[44] Y. Wang, Q. Chi, J. Zhang, N.S. Hush, J.R. Reimers, J. Ulstrup, J. Am. Chem. Soc. 2011, 133, 14856-14859.

[45] Q. Chi, O. Farver, J. Ulstrup, Proc. Nat. Acad. Sci. USA 2005, 102, 16203-16208.

[46] E.A. Della Pia, Q. Chi, D.D. Jones, J.E. MacDonald, J. Ulstrup, M. Elliott, Nano Lett. 2011, $11,176-182$.

[47] J. Zhang, A. Bilič, J.R. Reimers, N.S. Hush, J. Ulstrup, J. Phys. Chem. B 2005, 109, 1535515367.

[48] R.J. Nichols, S.J. Higgins, Acc. Chem. Res. 2016, 49, 2640-2648.

[49] T. Albrecht, A. Guckian, J. Ulstrup, J.G. Vos, Nano Lett. 2005, 5, 1451-1456

[50] Y. Li, H. Wang, Z. Wang, Y Qiao, J. Ulstrup, H.-Y. Chen, G. Chou, N.-J. Tao, Proc. Nat. Acad. Sci. USA 2019, 116, 3407-3412. 
[51] T. Albrecht, K. Moth-Poulsen, J.B. Christensen, J. Hjelm, T. Bjoernholm, J. Ulstrup, J. Am. Chem. Soc. 2007, 128, 6574-6575.

[52] J. Zhang, A.M. Kuznetsov, J. Ulstrup, J. Electroanal. Chem. 2003, 541, 133-146.

[53] Q. Chi, J. Zhang, P.S. Jensen, H.E.M. Christensen, J. Ulstrup, Faraday Discussions 2006, $131,181-195$.

[54] K. Johnsons-Winters, A.R Norstrom, S. Emesh, A.V. Astashkin, A. Rajapakshe, R.E. Berry, G. Tollin, J.H. Enemark, Biochemistry 2010, 49, 1290-1296

[55] A.M. Kuznetsov, J. Ulstrup, J. Chem. Phys. 2002, 116, 2149-2165.

TOC

Human sulfite oxidase ( $h \mathrm{SO})$ is a homodimeric two-domain enzyme, with a Mo catalytic cofactor and an electron-transfer heme $b_{5}$, switching between open and closed conformations. Cyclic voltammetry on $\mathrm{Au}(111)$-electrodes modified by $\omega$-amino-octanethiol monolayers gives welldefined $h \mathrm{SO}$ electrocatalysis. Constant current in situ STM displays sigmoidal tunnelling current/overpotential correlations and a 2-5\% coverage of molecular scale contrasts, assigned to individual functional $h \mathrm{SO}$ molecules.

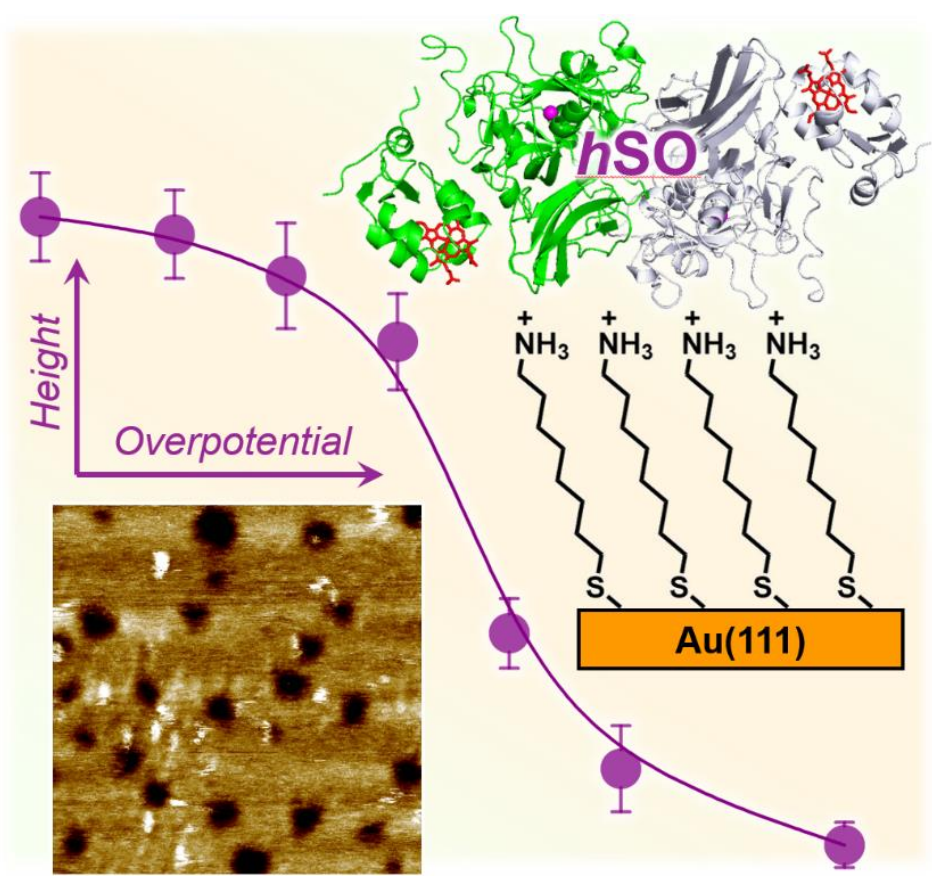

Prof. Jiawei Yan, Mr. Emil Egede Frøkjær, Dr. Christian Engelbrekt, Prof. Silke Leimkühler, Prof. Jens Ulstrup, Prof. Ulla Wollenberger, Dr. Xinxin Xiao, and Prof. Jingdong Zhang 
Voltammetry and Single-molecule in Situ Scanning Tunnelling Microscopy of the Redox Metalloenzyme Human Sulfite Oxidase

This article is protected by copyright. All rights reserved. 